\title{
Effect of Particle Gradation of Bed Material on Transport of Nonuniform Sediments
}

\author{
K.P.P. Pathirana, K.P.J.S. Kumara, T.B.S. Priyashantha and S.B. Darshana
}

\begin{abstract}
The effect of particle gradation on sediment transport rates of nonuniform sediment mixtures was investigated. The application of existing sediment transport formulae which are based on a single fixed grain diameter was found to be inappropriate to estimate transport rates for sediment mixtures. An equivalent sediment diameter defined by using a size gradation compensation factor $(\mathrm{Kg})$ relating the particle gradation and flow characteristics was used in the Engelund and Hansen formula to predict the transport rates of sediment mixtures. The formulation of the $\mathrm{Kg}$ factor was an improved version of the one suggested by Molinas and $\mathrm{Wu}[6]$ to extend the applicability of the method over a wide range of sediment sizes. The proposed method was tested against the data obtained from a series of laboratory experiments conducted over a movable bed with different sediment gradations. The significant improvement of computed sediment transport rates indicates the superiority of the improved formulation of the $\mathrm{Kg}$ factor in accounting for the effect of size gradation on transport rates. By correlating different non-dimensional parameters, a new relationship was found for $\mathrm{Kg}$, which could be readily applicable for sediment mixtures to compute transport rates over a wide range of sediment sizes.
\end{abstract}

Keywords: Nonunifrom sediments, Particle gradation, Bed load transport

\section{Introduction}

An accurate estimation of sediment transport in alluvial channels and rivers is important in hydraulic engineering, as it directly or indirectly influences many problems such as the prediction of natural changes of river morphology, the dredging of sediment deposits, the estimation of the lifetime of reservoirs and the prediction of pollutant transport etc. The critical condition of incipient motion and the transport mechanism of uniform sediments have been extensively investigated. The formulae developed by various researchers that are based on a single fixed representative grain diameter are widely used for estimating the bed load transport rates of uniform sediments (Einstein [3]; Meyer-Peter and Muller [5]). Natural river sediments are, however, non uniform and the transport of bed materials with these types of sediments are more complicated than that of uniform sediments. In the processes of non-uniform sediment movement, the fine particles on the bed are sheltered by coarse particles, leading to a relatively smaller transport rate. The situation is different for coarse particles on the bed as they are more exposed to the flow than if they were in a uniform sediment bed and hence, coarse particles are easier to be transported than the uniform sediment of equivalent sizes. It is, therefore, generally difficult to define the threshold condition for initiation of motion of a given size of grain in a sediment mixture and also its transport rate as they are highly influenced by the presence of other fractions in the mixture.

The most commonly used approach for estimating the transport rates of nonuniform sediments was based on the transport rates of individual fractions present in the sediment mixture and then taking the summation of the fractional transport rates. This method was found unrealistic for sediment mixtures, as it does not include the effect of different size fractions in the mixture on the motion of an individual grain size.

Various representative bed material sizes have been proposed for the transport formula developed for uniform bed material, where a single particle size is used. Einstein [3] recommended that D35 be used as the

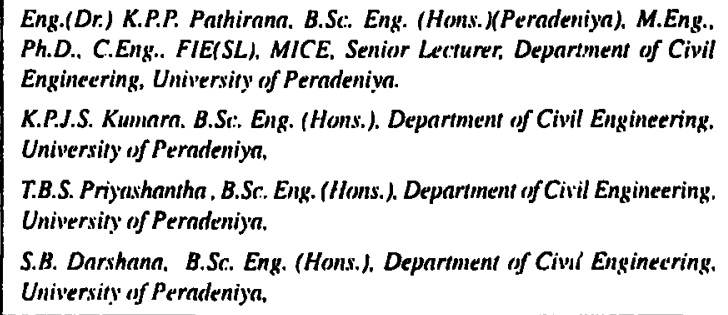


representative diameter of bed material for nonuniform sediment mixtures, where D35 stands for the diameter for which $35 \%$ of the bed material is finer. The weighted mean diameter of bed material was suggested by Meyer-Peter and Muller [5] in their equation for computation of transport rates of graded sediments. The effective diameter defined by Nordic [9] as, $d=1 /\left(\Sigma \Delta P_{b i} / d_{i}\right)$ was also recommended as the representative diameter of sediment mixtures, where, $\Delta P_{b i}$ is the fraction of bed material by dry weight, corresponding to the size fraction $i$ and $d_{i}$ is the diameter of bed material corresponding to the size fraction $i$. However, the use of a representative grain diameter in sediment transport formulae has not produced much improved results for many real life applications, suggesting that a single fixed size is not adequate to represent the various size fractions present in sediment mixtures.

In addition to the mean diameter, a sediment nonuniformity factor expressed by $d_{9 d} d d_{30}$ was proposed as a size distribution parameter for prediction of transport rates in steep slope rivers (Wu et al [11]). Misri et al. [8] and Samaga et al. [10] established empirical functions for estimating the fractional transport rates of the bed material load. However, several empirical constants are to be determined when using these methods and hence are less attractive in applications.

Molinas and Wu [6] presented a somewhat simplified approach for the computation of transport rates for sediment mixtures using a size gradation compensation factor as a measure of the effect of size gradation and flow intensity on the transport. This size gradation compensation factor was then combined with the mean diameter of the sediment mixture and an equivalent representative diameter was defined for the Engelund and Hansen [4] formula to compute the transport rates of sediment mixtures. However, this method has been verified only for fine sediment and hence it lacks generality.

As the method proposed by Molinas and Wu [6] was relatively easier to use even with the existing sediment transport formulae with little modification, it was decided to investigate this method further to make it applicable over a wide range of sediment sizes and flow conditions. In this paper, the size gradation compensation factor proposed by Molinas and $\mathrm{Wu}$ [6] was modified by incorporating the dimensionless unit stream function and the method was tested using laboratory data on the transport of nonuniform sediments with relatively coarse sediments. The test data collected from Samaga et al [10] was also used in the analysis to cover the different range of grain sizes. An attempt has been made to correlate the size gradation compensation factor with the parameters related to characteristics of bed material and flow intensity.

\section{Experimental Details}

The experiments were carried out in a $10 \mathrm{~m}$ long, $0.4 \mathrm{~m}$ wide, $0.5 \mathrm{~m}$ deep rectangular, recirculating, tilting flume in the Hydraulics Laboratory of the Faculty of Engineering, University of Peradeniya (Figure 1). A $2 \mathrm{~m}$ long stretch of the channel located at a distance of $4.5 \mathrm{~m}$ from the channel entrance was selected as the test section. The initial distance of $4.5 \mathrm{~m}$ length was found necessary to establish a fully developed flow in the channel, which was confirmed through a detailed analysis of measured velocity profiles along a fixed bed channel.

Natural river sand of relative density 2.65 was used in the experiments. Four sediment mixtures having different mean diameters and geometric standard deviations were used. Figure 2 shows the grain size distribution curves of sediment samples. The sediment used in the experiments was slightly larger than coarse sand. Once the channel was adjusted to a required slope and the sediment was placed in the channel, flow was passed over the movable bed. A sharp crested weir fixed at the end of the channel with a depth gauge was used to measure the flow discharges. Water depths were also recorded at several places along the test section using a depth gauge with an accuracy of $0.1 \mathrm{~mm}$. The transported materials were collected into a sediment trap located at the end of the channel. The weight of sediments accumulated in the trap was measured continuously using a load cell coupled with an $A D$ converter. A sediment feeder was used to supply the same bed material at the upstream end of the channel at a constant rate to maintain sediment equilibrium. For each sediment mixture, the test was repeated for a combination 


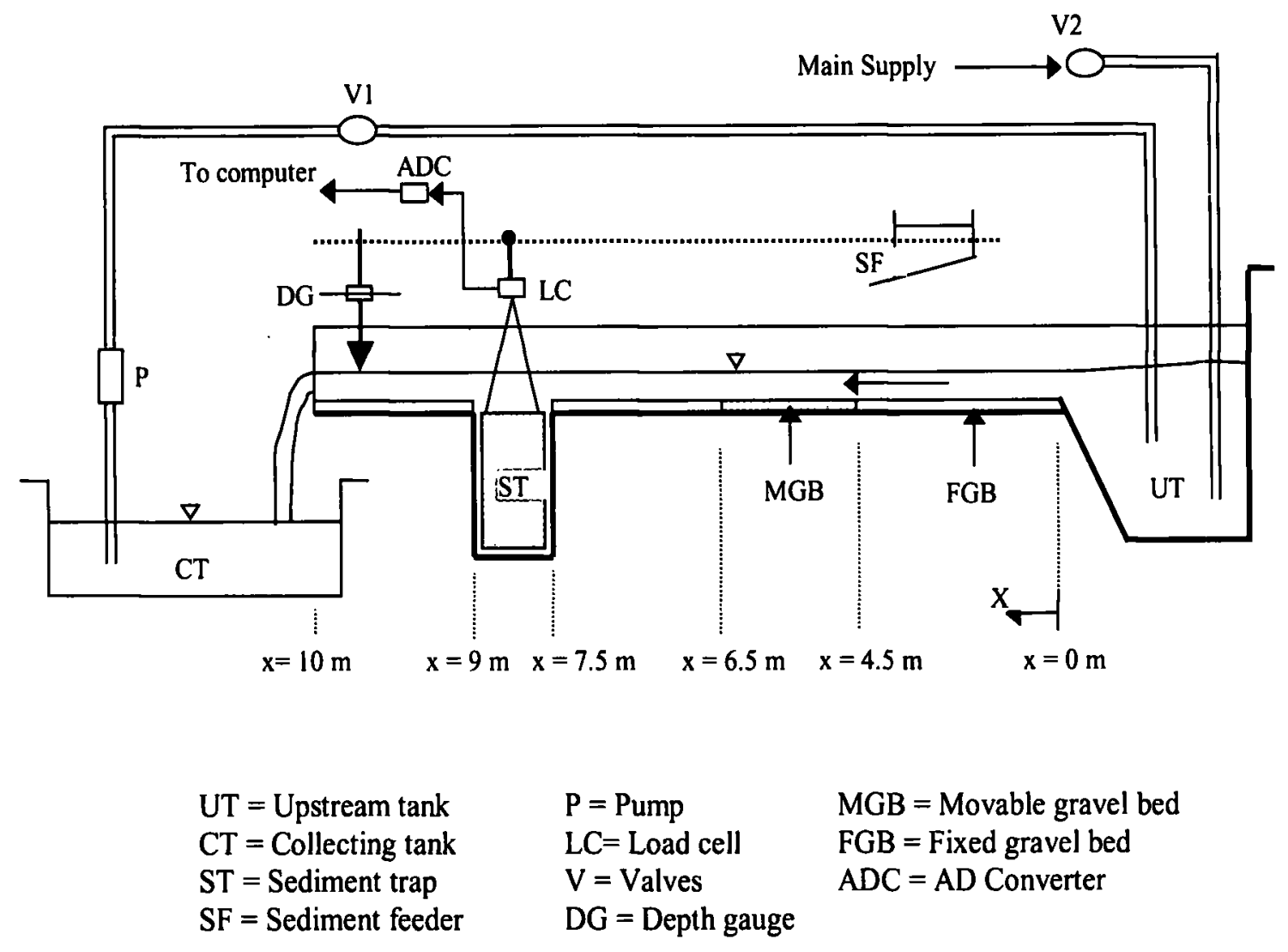

Figure 1 -.Experimental set-up

Table 1 - Summary of experimental data of present study.

\begin{tabular}{|c|c|c|c|c|c|c|c|}
\hline Run no: & \begin{tabular}{|c|} 
Median \\
diameter \\
$(\mathrm{mm})$
\end{tabular} & $\begin{array}{c}\text { Geometric } \\
\text { standard } \\
\text { deviation }\end{array}$ & Channel slope & $\begin{array}{c}\text { Discharge } \\
(1 / s)\end{array}$ & $\begin{array}{l}\text { Depth of } \\
\text { flow (m) }\end{array}$ & $\begin{array}{c}\text { Bed load } \\
\text { transport } \\
\text { rate }(\mathrm{N} / \mathrm{m} . \mathrm{s})\end{array}$ & $\begin{array}{l}\text { No of } \\
\text { data } \\
\text { sets }\end{array}$ \\
\hline G1 & 3.7 & 1.60 & $0.0055-0.0135$ & $8.0-24.0$ & $\begin{array}{r}0.040- \\
0.105\end{array}$ & $\begin{array}{r}0.088- \\
3.610\end{array}$ & 12 \\
\hline G2 & 4.3 & 1.31 & $0.0055-0.0135$ & $8.0-24.0$ & $\begin{array}{r}0.038- \\
0.108\end{array}$ & $\begin{array}{r}0.137- \\
2.953\end{array}$ & 12 \\
\hline G3 & 4.6 & 1.71 & $0.0055-0.0135$ & $8.0-24.0$ & $\begin{array}{r}0.045- \\
0.106\end{array}$ & $\begin{array}{r}0.049- \\
2.649\end{array}$ & 12 \\
\hline G4 & 5.1 & 2.05 & $0.0055-0.0135$ & $8.0-24.0$ & $\begin{array}{r}0.050- \\
0.110\end{array}$ & $\begin{array}{r}0.226- \\
3.247\end{array}$ & 12 \\
\hline
\end{tabular}

Table 2 - Summary of experimental data collected from Samaga et al. [10]

\begin{tabular}{|c|c|c|c|c|c|c|c|c|}
\hline $\begin{array}{c}\text { Run } \\
\text { no: }\end{array}$ & $\begin{array}{c}\text { Median } \\
\text { diameter } \\
(\mathbf{m m})\end{array}$ & $\begin{array}{c}\text { Geometric } \\
\text { standard } \\
\text { deviation }\end{array}$ & $\begin{array}{c}\text { Channel } \\
\text { slope }\end{array}$ & $\begin{array}{c}\text { Discharge } \\
(\mathrm{V} / \mathrm{s})\end{array}$ & $\begin{array}{c}\text { Depth of } \\
\text { flow (m) }\end{array}$ & $\begin{array}{c}\text { Total load } \\
\text { transport } \\
\text { rate (N/m.s) }\end{array}$ & $\begin{array}{c}\text { Bed load } \\
\text { transport } \\
\text { rate (N/m.s) }\end{array}$ & $\begin{array}{c}\text { No of } \\
\text { data } \\
\text { sets }\end{array}$ \\
\hline M1 & 0.35 & 2.60 & $0.005047-$ & $5.576-$ & $0.057-$ & $0.943-$ & $1.51-$ & 09 \\
& & & 0.00687 & 14.638 & 0.101 & 4.587 & 3.847 & \\
M2 & 0.29 & 2.36 & $0.006922-$ & $7.536-$ & $0.0692-$ & $1.389-$ & $1.083-$ & 09 \\
& & & 0.00996 & 14.359 & 0.0996 & 6.462 & 5.782 & \\
M3 & \multirow{2}{*}{0.27} & 2.17 & $0.005415-$ & $6.26-$ & $0.0562-$ & $1.879-$ & $1.535-$ & 09 \\
& & & 0.006097 & 13.38 & 0.0908 & 5.765 & 4.820 & \\
M4 & \multirow{2}{*}{0.20} & \multirow{2}{*}{1.44} & $0.004487-$ & $8.139-$ & $0.0618-$ & $1.820-$ & $1.241-$ & 06 \\
& & & 0.006097 & 13.459 & 0.0911 & 4.476 & 3.254 & \\
\hline
\end{tabular}




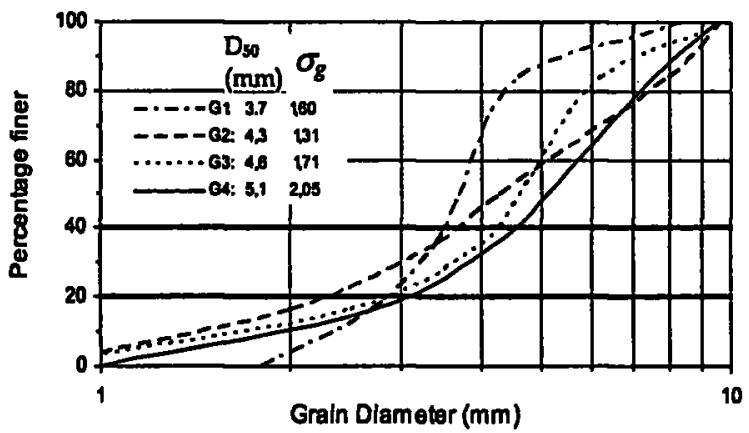

Figure 2- Grain size distribution of sediments

of three different discharges and four different channel slopes. A total of 48 test runs were carried out during this study and the range of test data is tabulated in Table 1.

No suspended sediment transport as well as the formation of bed undulations was observed in all these test runs. The experimental data of Samaga et al. [10] was also used in this study to investigate the influence of particle gradation on transport of sediment mixtures (Table 2). The sediment used in their experiments was medium to fine sand.

\section{Data Analysis}

\subsection{Effect of size gradation}

According to the fundamental relationships derived for the transport of bed load in steady, uniform flow conditions, the transport rate given in non-dimensional form $\left(\pi_{q b}\right.$ or $\left.\pi_{q b}^{\prime}\right)$ can be expressed as a function of dimensionless grain shear stress $\left(X_{2}\right)$. This was derived using the dimensional analysis and proved to be valid for uniform sediment particularly with coarse particles. The validity of this relationship was tested for nonuniform bed material using the data collected during this study. Two versions of the relationship used in this analysis are given below;

$\pi_{q b}=f\left(X_{2}\right)$ and $\pi_{q b}^{\prime}=f\left(X_{2}\right)$

where $\pi_{q b}=\frac{q_{b}}{\rho u_{.}^{3}}, X_{2}=\frac{\tau_{b}^{\prime}}{\gamma_{s} \cdot d}, \pi_{q b}^{\prime}=\frac{q_{b} \rho^{1 / 2}}{\left(\gamma_{s} d\right)^{3 / 2}}$ $\tau_{b}^{\prime}=\gamma R_{h}^{\prime} S$ and $\gamma_{s^{*}}=\left(\gamma_{s}-\gamma\right)$

Here, $\pi_{q b}$ and $\pi_{q b}^{\prime}=$ transport parameters, $d=$ median sediment diameter, $\mathrm{qb}=$ bed-load discharge, $\gamma_{\mathrm{s}}$ and $\gamma=$ the specific weight of sediment and water, respectively, $R_{h}^{\prime}=$ hydraulic radius due to grain roughness and $S=$ slope of energy line.

Figure 3 shows the data plotted on a graph of transport parameters against the dimensionless grain shear stress. Although a good correlation is observed between $\left(\pi_{q b}\right.$ and $\left.\pi_{q b}^{\prime}\right)$ and $X_{2}$ for the data presented here, a considerable scatter exists around the regression equations particularly for the sediment mixtures whose median grain sizes are coarse. This can be partly due to the gradation of sediment mixtures used for bed material.

The degree of scatter is comparatively less with the data of Samaga et al.[10] compared with the present experimental data and this could be mainly due to the use of medium to fine sediments in their tests. This suggests that the hiding and exposure effect of sediment grains in nonuniform sediment mixtures is much more pronounced when the mixture is having a wide size gradation ranging from medium sand to coarse granular material. This has also been highlighted by Wu et al. [12] in their studies on sediment mixtures. To account for the influence of bed material gradation, they introduced a hiding and exposure factor which is stochastically related to the size and gradation of bed materials to modify the criteria for the sediment incipient motion proposed by Shield (Chang [1]). However, this approach is not easy to apply and thus, is less attractive in applications.

The experimental data has also been tested with the well known relationship proposed by Einstein (Chang [1]) where two nondimensional parameters known as the Einstein bed load parameter $\phi$, and Einstein flow intensity parameter $\psi$, were used to represent steady state sediment transport. These parameters are given as;

$\phi=\frac{q_{b}}{\gamma_{s}}\left[\frac{\gamma}{\left(\gamma_{s}-\gamma\right) g d^{3}}\right]^{1 / 2}$

and

$\psi=\left(\frac{\gamma_{s}-\gamma}{\gamma}\right) \frac{d}{R_{h}^{\prime} S}$

Figure 4 illustrates a comparison between the experimental data with the Einstein bed load 
function. The data collected from Samaga et al. [10] has also been plotted on the same graph. It can be seen from the figure that the data points are highly scattered for the sediment mixtures with coarse grains. The parameters, $\phi$ and $\psi$ computed using the test data are somewhat smaller than those predicted by the Einstein function.

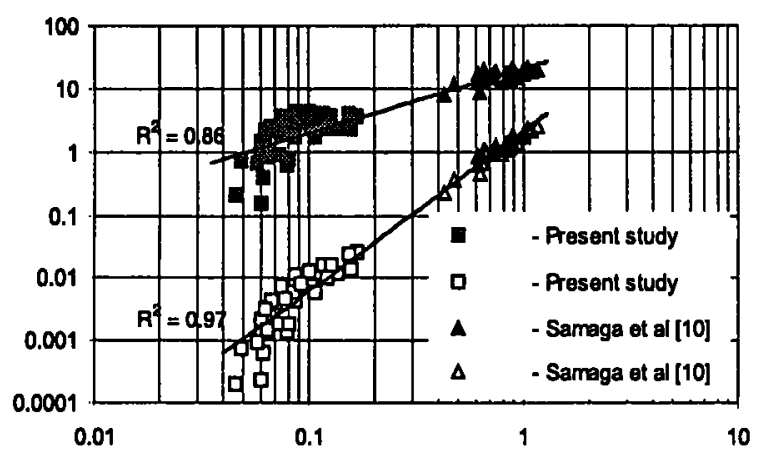

Figure 3 - Relationship between transport paraneter and dimensionless grain shear stress.

Several other transport formulae reported in the literature were also used for comparison with the experimental data and a similar behaviour was found with test data generally scattered around the formulae used. This highlights the fact that the transport phenomenon of bed material is more complicated with non uniform sediment mixtures than that with uniform sediment. In order to improve the predictive capability of bed load transport rates of nonuniform bed material, the use of variable representative grain sizes was analysed in detail as that would better represent the sediment mixture than only a fixed particle diameter such as, $D_{35}$ or $D_{50}$ of bed material. Description of the proposed method applied to Engelund and Hansen [4] is given in the following section.

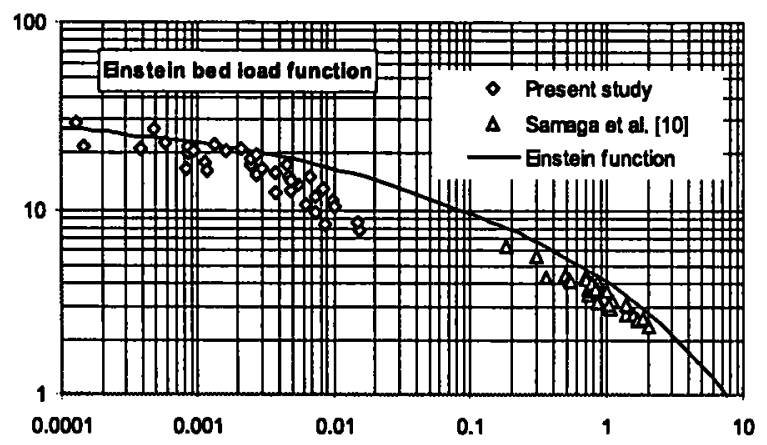

Figure 4 - Comparison between experimental data with the Einstein bed load function.

\subsection{Size gradation compensation factor}

Molinas and Wu [6] developed a size gradation compensation factor, which is based on the size gradation and the flow intensity, to modify the median diameter of sediment as a variable representative size to be used in the Engelund and Hansen formula for estimating the sediment transport rates of nonuniform sediment mixtures. In this study, this method was further analysed with sediment mixtures having coarse particles.

The transport formula proposed by Engelund and Hansen [4] takes the following form,

$f^{\prime} \phi=0.1 \theta^{2.5}$

where, $\phi=\frac{q_{s}}{\gamma_{s} \sqrt{\frac{\gamma_{s}-\gamma}{\gamma} g D^{3}}}$

$\theta=\frac{\tau}{\left(\gamma_{s}-\gamma\right) D}$

$f^{\prime}=\frac{2 g S h}{V^{2}}$

in which, $f^{\prime}=$ friction factor, $V=$ average flow velocity, $D=$ sediment size, $\theta=$ dimensionless shear stress, $\phi=$ dimensionless sediment transport function, $\tau=$ shear stress along the bed, $q_{s}=$ total bed material discharge by dry weight per unit width.

Figure 5 shows a comparison of the experimental data with the Engelund and Hansen formula and the test data of Samaga et al. [10] is also presented on the same graph. Please note that the data set M3 of Samaga et al. [10] was not used in the analysis and it was reserved for verification of the proposed method in the latter

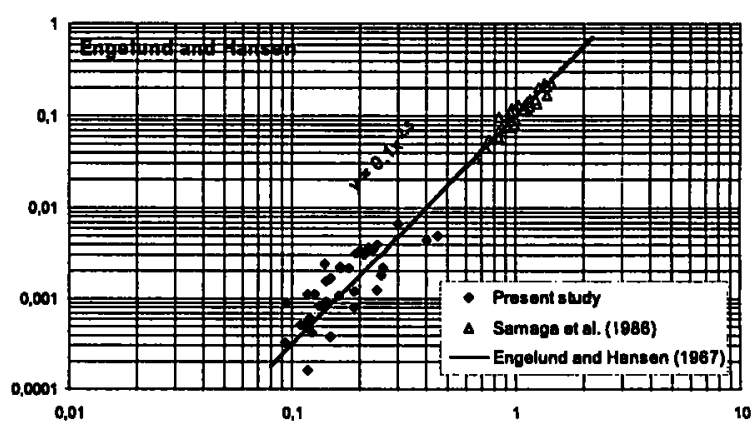

Figure 5 - Relationship between $f^{\prime} \phi$ and $\theta$ for the experimental data of Samaga et.al, [10]. 


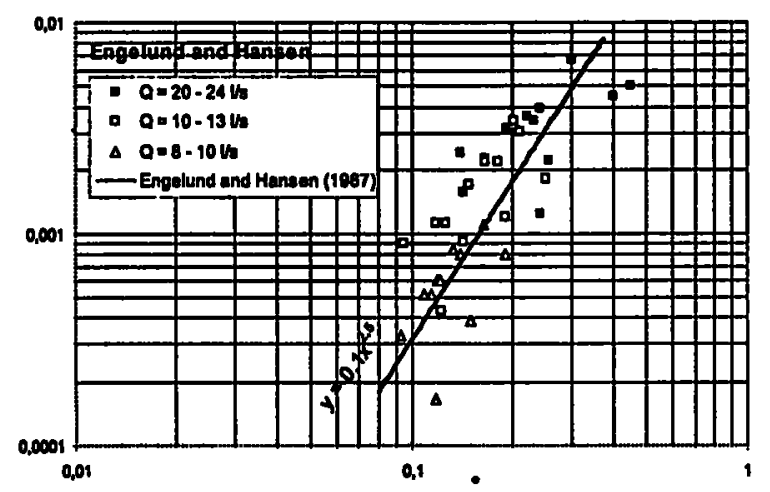

Figure 6- Relationship between $f^{\prime} \phi$ and $\theta$ for the experinental data sorted by water discharge.

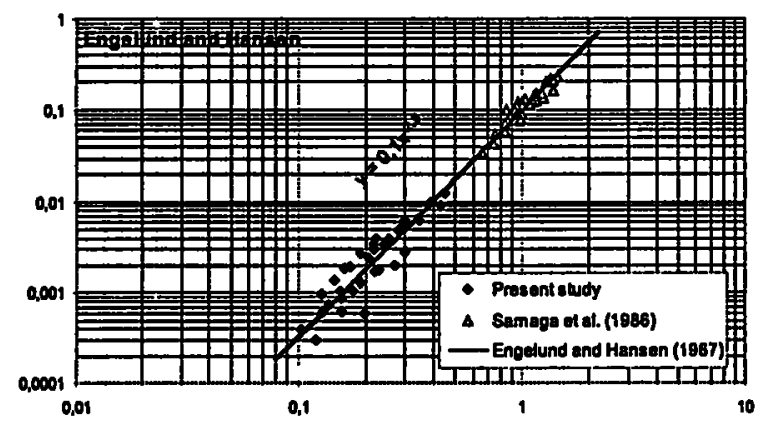

Figure 7- Relationship between $f^{\prime}(\phi)$ and $\theta$ for the experimental data with $K_{8}$ factor.

part of this study. The grain sizes used in the formula were $D_{50}$ of different sediment mixtures. Although the data points approximately follow the Engelund and Hansen formula, a considerable scatter is shown around the theoretical line. A high degree of scatter is seen with the sediment mixtures whose median grain sizes are very coarse. This may be partly due to the particle gradation of sediment mixtures.

The experimental data was plotted on a graph of $f^{\prime}(\phi)$ versus $\theta$ for three ranges of water discharges as shown in Figure 6. It can be clearly seen that the degree of scatter is higher for the test cases conducted with a high discharge range than that with the low discharge range. This implies that if any adjustment factor is to be introduced to modify the median diameter of sediment particles to improve model predictions, this factor should not be a fixed one having a single value for all the test cases, but should vary from one run to another according to the flow parameters as well as particle gradation. Accordingly, an equivalent sediment diameter, $D_{c}$ is proposed as;

$D_{r .} K_{g} D_{s o}$ where, $K_{f}$ is the size gradation compensation factor which depends on the flow intensity and particle gradation of sediment mixtures.

Molinas and Wu [6] presented a functional relationship for the $K_{\delta}$ factor as similar to Eq.(9), but $\left(u_{*} / w_{50}\right)$ was used in their formulation instead of the dimensionless unit stream function $\left(V S / w_{50}\right)$ where, $u_{\text {. }}=$ shear velocity, $S=$ channel slope and $w_{50}=$ fall velocity of grains corresponding to size $D_{50}$. In this study, the $K_{\mathrm{g}}$ factor is related to the dimensionless unit stream function $\left(V S / w_{50}\right)$ and $\sigma_{g}$ as given in Eq. (9). The inclusion of $\left(V S / w_{50}\right)$ in place of $\left(u . / w_{50}\right)$ is mainly for a better representation of the flow intensity on the transport phenomenon.

$$
K_{g}=\frac{A}{1+B\left(V S / w_{50}\right)^{\alpha}\left(\sigma_{g}-1\right)^{\beta}}
$$

in which, $\sigma_{g}=$ geometric standard deviation of sediment mixture, where, $\sigma_{8}=\sqrt{D_{4} / D_{16}}, D_{84}$ and $D_{16}=$ particle sizes for which $84 \%$ and $16 \%$ of grains are finer by dry weight, respectively. $A, B$, $\alpha$ and $\beta$ are coefficients.

The experimental data shown in Figure 5 was plotted again in Figure 7 after incorporating the $K_{\mathrm{g}}$ factor to modify the median diameter of sediment in the transport formula. The results seem to be encouraging as the degree of scatter reduces with the application of the $K_{\mathrm{g}}$ factor, indicating that the effect of particle gradation on transport rates are somewhat eliminated. The scattering of data still present could be due to various other effects influencing the transport phenomenon including experimental errors. To compare the computed sediment transport rates with the measured data, two statistical parameters were used and these parameters are given as;

a) The standard deviation between the computed and measured transport rates:

$\sigma=\sqrt{\frac{\sum_{i=1}^{N}\left(q_{i c}-q_{i m}\right)^{2}}{N-1}}$

where, $q_{i c}$ and $q_{i m}=$ computed and measured transport rates in $\mathrm{kg} / \mathrm{m} . \mathrm{s}$, respectively and $N=$ total number of data sets. 
b) The mean normalized error:

$$
M N E=\frac{100}{N} \sum_{i=1}^{N}\left|\frac{q_{i c}-q_{i m}}{q_{i m}}\right|
$$

A comparison between the computed and measured transport rates for three different test cases are given in Table 3. Use of the median diameter as the sediment size in the Engelund and Hansen [4] formula was taken as the first test case while the application of variable representative size defined according to the Eqs.(8) and (9) was considered for the second test case. The method proposed by Molinas and $\mathrm{Wu}$ [6] for the representative particle diameter using the $K_{g}$ factor expressed in terms of $\left(u_{*} / w_{50}\right)$ and $\sigma_{g}$ was used as the third case.

It can be clearly seen that the standard deviation and mean normalised error between computed and measured bed load transport rates are reduced once the $K_{\mathrm{g}}$ factor is used. The reduction of these statistical parameters is much more pronounced for the second test case than that for the third case, particularly for the experimental data obtained during this study. This implies that the $K_{\mathrm{g}}$ factor given in Eq.(9) is able to produce much better results than the factor proposed by Molinas and Wu [6], particularly for sediment mixtures with relatively coarse particles. Approximately the same transport rates are predicted for the test cases 2 and 3 with the data of Samaga et al. [10], suggesting that the $\mathrm{Kg}$ factor given in Eq.(9) and the one proposed by Molinas and Wu [6] are equally good for sediment mixtures with fine grains.
To obtain a better correlation between computed and measured transport rates, the coefficients used in Eq.(9) were not the same for two sets. of data used in this analysis. Probably this may be attributed to the use of very different sediment sizes used in these experiments. Table 4 gives the values of the coefficients used in Eq.(9) for calculating the $K_{\mathrm{g}}$ factor for different data sets.

Depending on the particle gradation, if different values are used for the coefficients in Eq.(9) to compute the $K_{\mathrm{g}}$ factor, then one may find it difficult to apply this method for a new sediment mixture without knowing the values of these coefficients. However, it is interesting to note that a better correlation exists between $\left(X_{2}\right.$ $\left./ K_{\mathrm{g}}\right)$ and $\left(h\left(\sigma_{\mathrm{g}}-1\right) / D_{50}\right)$ when using the complete data set as shown in Figure (8), where, $X_{2}=\gamma R_{h}^{\prime} S / \gamma_{s} . D_{50}$

Although the data points are somewhat scattered, it clearly shows an increasing trend of $\left(X_{2} / K_{\mathrm{g}}\right)$ with $\left(h\left(\sigma_{\mathrm{g}}-1\right) / D_{50}\right)$ and asymptotically reaching a constant value when this is larger than about 400 . It can be seen from Figure 8 that for a given bed material size and

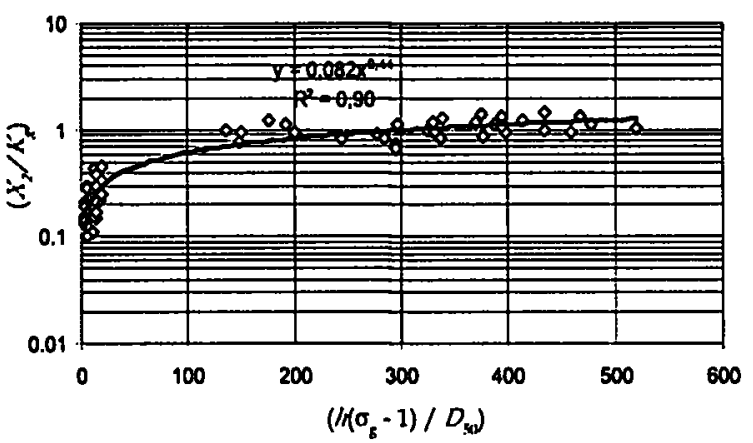

Figure 8 - Relationship between $\mathrm{X}_{1} / \mathrm{K}_{3}$ and $\left(h(\sigma g-1) / D_{50}\right)$

Table 3 - Summary of comparison between computed and measured sediment transport rates for different test cases. (The values indicated within brackets in third and fourth columns are \% reduction of and MNE)

\begin{tabular}{|l|l|l|l|}
\hline \multicolumn{1}{|c|}{ Test case } & \multicolumn{1}{|c|}{ Data used for the analysis } & $\begin{array}{c}\text { Standard } \\
\text { deviation ( } \sigma) \\
(\mathbf{k g} / \mathbf{m . s})\end{array}$ & $\begin{array}{c}\text { Mean } \\
\text { normalised } \\
\text { error, MNE (\%) }\end{array}$ \\
\hline 1. Using D50 (without $K_{\mathrm{g}}$ ) & Present study (Table 1) & 9.7 & 52 \\
& Samaga et al. [10] (Table 2) & 14.3 & 35 \\
2. Using De (with $K_{\mathrm{g}}$ as per & Present study (Table 1) & $3.8(61 \%)$ & $38(27 \%)$ \\
Eq.(9)) & Samaga et al. [10] (Table 2) & $5.5(62 \%)$ & $12(66 \%)$ \\
3. Using De (with Kg as per & Present study (Table 1) & $5.2(46 \%)$ & $43(17 \%)$ \\
Molinas and Wu [6]) & Samaga et al. [10] (Table 2) & $5.8(59 \%)$ & $13(63 \%)$ \\
\hline
\end{tabular}


Table 4 - The values of coefficients used in Eq.(9).

\begin{tabular}{|l|c|c|c|c|}
\hline $\begin{array}{l}\text { Data used for } \\
\text { the analysis }\end{array}$ & A & B & $\alpha$ & $\beta$ \\
\hline Present study & 1.2 & 0.8 & 0.1 & 0.6 \\
\hline Samaga et al. [10] & 0.9 & 0.2 & 0.1 & 1.5 \\
\hline
\end{tabular}

flow characteristics, for larger gradations, $\left(h\left(\sigma_{g}\right.\right.$ - 1) / $D_{50}$ ) will be larger and the corresponding values of $K_{\mathrm{g}}$ will be smaller resulting in higher transport rates. This can be expected as the finer fraction of highly graded sediment mixtures are more readily transported leading to high transport rates. The reverse is also true, for smaller gradations, $\left(h\left(\sigma_{g}: 1\right) / D_{50}\right)$ will be smaller resulting in larger $\mathrm{Kg}$ values and which then, predict smaller transport rates. On the other hand, for a given particle gradation and flow characteristics, if the median particle diameter is large, $\left(h\left(\sigma_{\mathrm{g}}-1\right) / D_{50}\right)$ will be smaller and hence, $K_{\mathrm{g}}$ values will be larger and that leads to smaller transport rates. This is also expected in that larger diameter particles are not easily transportable and as a result, transport rates will be lower.

So, it appears that the functional relationship of the $K_{\mathrm{g}}$ factor given in Figure (8) is able to generate more sensible values for computing representative sediment diameters for Eq.(8). Therefore, Eq.(12) can now be used directly for estimating $K_{\mathrm{g}}$ values by knowing the size gradation of bed material and flow characteristics instead of using Eq.(9) that eliminates the prior knowledge of various coefficients used in Eq.(9). In addition, Eq.(12) can also be applicable for a wide range of sediment gradations whose median diameter of grains vary from granular material to very fine sand.

$K_{8}=12.2 X_{2}\left[\frac{h\left(\sigma_{8}-1\right)}{D_{50}}\right]^{-0.44}$, for $\sigma_{g}>1$

Figure (8) also shows that for a given particle gradation, when the median diameter of grains is progressively reduced, the term $\left(h\left(\sigma_{g}-1\right) /\right.$ $D_{50}$ ) will be increased gradually and eventually reach a constant value of $\left(X_{2} / K_{g}\right)$ when $\left(h\left(\sigma_{g}-\right.\right.$ 1) $\left./ D_{50}\right)$ is larger than about 400 . This indicates that the $\mathrm{Kg}$ factor will not be influenced by particle gradation for larger values of. $\left(h\left(\sigma_{g}-1\right)\right.$ $/ D_{50}$ ) and will depend only on $X_{2}$. Therefore, the effect of bed material gradation on sediment transport is not much pronounced when the sediment diameter is very fine. This has been noticed previously in Sec.(3.1) when the experimental data plotted in Figures (3) and (4) are discussed.

\section{Verification}

It is essential that the method proposed in this study to estimate the sediment transport rate of non uniform sediment be verified against laboratory and field data before it is recommended for real life applications. Unfortunately a completely independent data set was not available for this purpose. However, as a preliminary verification of the proposed method, it was decided to use the M3 data set of Samaga et al [10], which was not used for the data analysis. This data set comprised nine test runs conducted for a sediment mixture having a median diameter of grains of $0.27, \mathrm{~mm}$ and geometric standard deviation 2.17. The hydraulic parameters relevant to these tests are given in Table 2. Figure (9) shows a comparison between computed and measured sediment transport rates with and without the $\mathrm{Kg}$ factor proposed in Eq.(12).

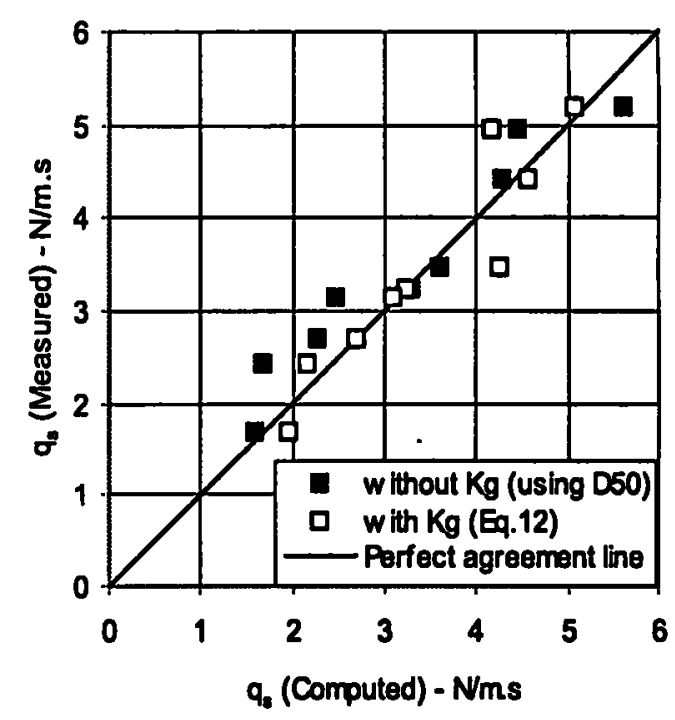

Figure 9 - Comparison between computed and measured sediment transport rates using M3 data set of Samaga et.al. [10] with and without $\mathrm{Kg}$ factor.

The data points are slightly scattered when using the median diameter of the grains as the sediment size in Engelund and Hansen [4] as shown in Figure (9). The application of the size gradation compensation factor $\left(K_{\mathrm{g}}\right)$ proposed in Eq.(12) for modifying the grain size has resulted in much improved results. The standard 
deviation between computed and measured transport rates is reduced from $8 \%$ to $4 \%$ when the computations are done without and with the use of the $K_{\mathrm{g}}$ factor, respectively. In addition, the mean normalized error is also reduced from $20 \%$ to $8 \%$ in the analysis without and with the use of the $K_{\mathrm{g}}$ factor, respectively. Although the above test appears to give very positive results, a proper verification has to be done using a completely independent data set. Besides, the method should also be tested for a wide range of sediment mixtures and flow conditions using laboratory and field data. However, the results shown above is highly encouraging and hence, it is worthwhile to explore the proposed method further as it is a very simplified approach to estimate the transport rates of nonuniform sediment and is easy to use in real applications.

\section{Summary and Conclusions}

A series of laboratory experiments was conducted to investigate the effect of particle gradation on sediment transport rates of nonuniform sediment mixtures. The use of a single fixed size of sediment in existing transport formulae is proved to be not appropriate for estimating transport rates with non uniform bed materials as the results are highly scattered. The degree of scatter increases with the flow intensity and the size gradation of sediments.

In this study, the method proposed by Molinas and $W u$ [6] using the size gradation compensation factor $\left(K_{\mathrm{g}}\right)$ to compute the equivalent representative sediment diameter was modified using the dimensionless unit stream function together with the geometric standard deviation of sediment mixtures (Eq.9). This formulation of $K_{\mathrm{g}}$ was first tested as an interim measure to treat the effect of the particle gradation of bed material on transport rates. The equivalent representative diameter, $D$, obtained using this factor, was applied as the sediment diameter in the Engelund and Hansen [4] formula to estimate the sediment transport rates. The method was tested using the laboratory data and the data collected from Samaga et al. [10]. The sediments used for the laboratory experiments were relatively coarse and belong to the range of fine gravel to medium sand whereas, the data of Samaga et al. [10] were related to medium to fine sand, that made it possible to test the proposed method over a wide range of sediment particles. The agreement between the measured and computed transport rates with the proposed $K_{\mathrm{g}}$ factor was much better than that suggested by Molinas and Wu [6], particularly for sediment mixtures with coarse particles. However, both methods were able to estimate approximately the same transport rates for sediment mixtures with fine sediments.

A good correlation was obtained between $\left(X_{2} /\right.$ $\left.K_{g}\right)$ and $\left(h\left(\sigma_{g}-1\right) / D_{50}\right)$, which can be applicable over a wide range of sediment sizes and thereby, a new formulation was found for the $K_{\mathrm{g}}$ factor. The newly developed $K_{\mathrm{g}}$ factor (Eq.12) was verified against the limited data available and was found to give much improved results. This suggests that the new $K_{\mathrm{g}}$ factor is able to eliminate the effect of size gradation on sediment transport rates up to a certain extent. The proposed formula of the size gradation compensation factor needs to be further tested using more laboratory and field data to enhance the accuracy and reliability of the method and also to broaden the applicability of the method to a wide range of sediments.

\section{Acknowledgments}

The paper was drafted during the first author's associateship visit to the Abdus Salam Int. Center for Theoretical Physics (ICTP), Trieste, Italy in Feb. 2006 and hence, the assistance of the ICTP as well as the Swedish International Development Cooperation Agency (SIDA) who sponsored the associateship is gratefully acknowledged.

\section{References}

1. Chang, H.H., "Fluvial Processes in River Engineering", John Wiley and Sons, Inc., 1988.

2. Chien, N.Z. Wan, "Mechanics of Sediment Transport", The American Society of Civil Engineers, ASCE Press, p.395, 1998.

3. Einstein, H.A., "Bed Load Transport in Mountain Creek", Tech. Paper, No. 55, Soil Conservation Service, U.S. Dept. of Agr., Washington, D.C., 1944.

4. Engelund, F. and Hansen, E. A., "Monograph of Sediment Transport in 
Alluvial Streams", Tekinisk Forlag, Coheỉhlagen, Denmark, 1967.

5. Meyer-Peter, E. and Muller, R., "Formula for Bed Load Transport", Proc. 2nd Meeting, Vol.6, Int. Asn. Hydr. Res., Stockholm, pp.3964, 1948.

6. Molinas, A. and Wu, B., "Effect of Size Gradation on Transport of Sediment Mixtures", J. Hydr. Engrg., ASCE, 124(8), pp.786-793, 1998.

7. McEwan, I., Sorensen, M., Heald, J., Tait, S., Cunningham, G., Goring, D. and Willetts, B., "Probabilistic Modelling of Bed-Load Computations", J. Hydr. Engrg., ASCE, 130(2), pp.129-139, 2004.

8. Misri, R.L., Garde, R.J. and Ranga Raju, K.G., "Bed Load Transport of Coarse Nonuniform Sediment", J.' Hydr. Engrg., ASCE, 110(3), pp.312-328, 1984.
9. Nordic, C.F., "Application of Engelund and Hansen Sediment Transport Equation in Mathematical Models", Fourth Int. Symp. on River Sedimentation, China, pp.611-616, 1989.

10. Samaga, B.R., Ranga Raju, K.G. and Garde, R.J., "Bed Load Transport of Sediment Mixtures", J. Hydr. Engrg., ASCE, 112(11), pp.1003-1018, 1986.

11. Wu, B., Molinas, A. and Julien, P.Y., "BedMaterial Load Computations for Nonuniform Sediments" f. Hydr. Engrg., ASCE, 130(10), pp.1002-1012, 2004.

12. Wu, W., Wang, S.S.Y. and Jia, Y., "Nonuniform Sediment Transport in Alluvial Rivers", J. Hydr. Res., 38 (6), pp.427$434,2000$.

13. Yang, S., "Prediction of Total Bed Load Discharge", J. Int. Ass. Hydr. Eng. and Res., pp.12-22, 2005. 